Aim of the study: This study aimed to prospectively investigate the clinical outcomes of curative radical helical tomotherapy (HT) applied to recurrent non-small cell lung cancer (NSCLC) at the bronchial stump site after right pneumonectomy. After right pneumonectomy, the heart shifted right laterally. The chambers of the heart closed with a recurrent mass at the bronchial stump were the right atrium and left atrium due to right shifting of the heart. The unfavorable bronchial stump recurrent cancer-heart geometry due to a right shift of the heart might serve as a reliable predictor of cardiac morbidity for aggressive radiotherapy.

Material and methods: The 23 patients received HT for the recurrent NSCLC at the bronchial stump site after right pneumonectomy between 2008 and 2011. The median age of the patients was 65 years (range $56-74$ ).

Results: We prescribed $95 \%$ volume of the primary planning target volume (PTV) to a total dose of $69 \mathrm{~Gy}$ in $30 \mathrm{frac}-$ tions, and $95 \%$ of the secondary PTV to a total dose of $54 \mathrm{~Gy}$ in 30 fractions with reduction of the $50 \%$ volume of the heart < $20 \mathrm{~Gy}$. The median conformal index in the 23 plans was 1.21. The mean fraction of primary PTV receiving more than $95 \%$ of the prescribed dose was $97.8 \%$. The mean $\mathrm{V}_{45}, \mathrm{~V}_{50}, \mathrm{~V}_{60}$ of the heart were $10.5 \%, 6.5 \%, 0.2 \%$, respectively. The median follow-up after tomotherapy was 19.86 months. Median survival was 20 months. The 2-year OS was $39.1 \%$

Conclusions: The relatively high dose tomotherapy alone for patients with a recurrent bronchial stump mass which was proximal to the heart demonstrated favorable clinical results without severe heart or pulmonary complications.

Key words: recurrent NSCLC at right bronchial stump, heart, helical tomotherapy.

Contemp Oncol (Pozn) 2015; 19 (1): 60-71 DOI: $10.5114 /$ wo.2015.48179

\section{Preliminary results of tomotherapy for treatment of inoperable recurrent non-small cell lung cancer at bronchial stump site after right pneumonectomy}

Hong Seok Jang', Seong Kwon Moon ${ }^{2}$

${ }^{1}$ Department of Radiation Oncology, Medical College, Catholic University, St. Mary's Hospital, Seoul, South Korea

2Department of Radiation Oncology, Medical College, Soonchunhyang University, Bucheon Hospital, Seoul, South Korea

\section{Introduction}

Most patients suffering from non-small cell lung cancer (NSCLC) are advised to undergo surgery, especially if the ailment is in the early stages, including sometimes patients with stage IIIA disease.

Analysis of failure patterns in the past has indicated that loco-regional recurrence is a well-documented event in these cases, and occurrence of systemic failure in completely resected patients usually changes them into the incurable state [1, 2]. Many studies have been carried out in the field of local regional recurrence. These studies reveal that this recurrence ranges from 3 to $9 \%$ in stage I, and between 29 and 38\% in stage IIIA, depending on whether the patients underwent adjuvant treatment. After administering curative resection, 5-year survival can range from 10 to $21 \%$ among persons suffering from stage III adenocarcinoma, or between 54 and $84 \%$ for stage I squamous cell carcinoma (SCC) [3-5]. There are more cases of documented distant treatment failures after surgery than intrathoracic ones.

Persons who suffer from intrathoracic recurrence are mainly treated with endobronchial irradiation, completion pneumonectomy, and other aggressive approaches. Photodynamic therapy has also been shown to be quite ineffective among NSCLC patients suffering from inoperable recurrent NSCLC at the stump site $[6,7]$. According to a study conducted by Furukawa et al. [8], 75\% of patients who had suffered from NSCLC recurrence at the stump site experienced re-recurrence after undergoing photodynamic therapy, in spite of an initial positive response. However, other studies conducted in this field have more than contradicted this position. There are various studies which have shown that radiation therapy can be effective in treating lung cancer stump recurrence, even when it is not used in conjunction with other therapies $[9,10]$. All these studies were carried out over a long period of time and involved recurrence at several different sites, including at the bronchial stump, chest wall, and lymph node. These studies used diverse samples of patients who were treated using different doses and fractionation patterns. Additionally, external radiotherapy was at times used in combination with brachytherapy or chemotherapy. In this regard, the studies did not portray a comprehensive picture on the effectiveness of radiotherapy in disease treatment. This is regardless of the fact that some of them acknowledged that external beam radiation therapy (EBRT) was as effective as surgery.

In NSCLC, pneumonectomy is required in the removal of lesions which involve the bronchi. In the case of lung cancer, pneumonectomy is associated with significant mortality and morbidity rates, including lung edema, post- 
pneumonectomy syndrome, and bronchopleural fistula [11, 12]. However, in most cases, this cannot be avoided due to technical and anatomical considerations. In addition to changing pulmonary hemodynamics, pneumonectomy results in the migration of large vessels, as well as the heart, in the pneumonectomy cavity. This process takes place over a period of many years after one undergoes resection. After a patient undergoes right pneumonectomy, the heart will shift laterally. However, it is noted that in the case of left-sided pneumonectomy, the heart rotates in the cavity $[13,14]$.

There are many benefits associated with radiation therapy. For example, radiation therapy improves both overall survival and local control in stump recurrence after pneumonectomy is carried out. However, the survival benefits that result from secondary metastasis prevention and better local control can be offset by radiation-induced heart disease $[15,16]$. There are diverse explanations for this. One of them is the lateral shift of the heart after the patient suffering from lung cancer has undergone right pneumonectomy [17]. There are other ailments that a person may suffer from later in life as a consequence of cardiac irradiation caused by radiotherapy of a right recurrent bronchial stump tumor close to the right-shifted heart after right pneumonectomy. These include valvular disease, cardiomyopathy, and pericardial disease. Lung cancer patients who are also afflicted by different heart ailments have an increased risk of myocardial infarction or even death as compared to those patients without additional heart conditions. There are many contributing factors in this case. However, the main ones are cardiac volume and radiation dose $[18,19]$. In this study, it is supposed that extremely unfavorable bronchial stump recurrent cancer-heart geometry which is caused by the lateral shift of the heart (a heart proximal to the bronchial stump recurrent cancer) might serve as a reliable predictor of cardiac morbidity in patients with disadvantageous cardiac anatomy and localization for 3-dimensional radiation techniques. Tomotherapy has been found to significantly reduce the radiation dose delivered to the cardiac region and other uninvolved tissues among patients with left-sided breast cancer [20,21]. The main objective in such a case is to ascertain the effectiveness of tomotherapy in increasing the tumor dose, while at the same time reducing irradiation of the heart, the left lung and other tissues. Our present study concerns patients who suffer from inoperable recurrent NSCLC at the stump site after right pneumonectomy. In this study, we sought to analyze the preliminary results of tomotherapy for the treatment of inoperable recurrent NSCLC at the stump site after right pneumonectomy. The treatment was carried out by escalating the radiation dose to the tumor and reducing radiation exposure to the right-shifted heart and to the single remaining left normal lung due to right pneumonectomy.

\section{Material and methods}

\section{Study design}

The current study was a clinical trial that aimed at gathering preliminary results of tomotherapy for the treatment of inoperable recurrent NSCLC at the stump site after right pneumonectomy. From 2008 to 2011, 23 patients received curative radical tomotherapy for a recurrent stump mass. The results were gathered by escalating the radiation dose and reducing radiation exposure to the right-shifted heart and to the single remaining left normal lung due to right pneumonectomy. Patients who had received induction chemotherapy were not included in the study. The development, review, and management of the trial were carried out according to the international standards that govern human experimentation. The Hospital review board approved of the study before inclusion of any of the $23 \mathrm{pa}$ tients. All the patients consented to the trial in writing before they were included.

\section{Patient eligibility}

The patients used in the study were histologically proven to have stage I-III NSCLC. They were only eligible if it was possible to encompass the entire detectable tumor under the tomotherapy radiation field. The detectable tumor referred to included the primary tumor and lymph nodes that were involved. Patients with supraclavicular metastasis in the nodes were also included. All the patients were more than 18 years of age and had a performance status of more than or equal to $70 \%$. Because the interest of the study was to independently evaluate the efficiency of tomotherapy in dose escalation to the targeted tumor and toxicity reduction, patients undergoing concurrent chemotherapy were not included in the study. Other patients who were ineligible were those who had undergone other kinds of resections that were less than a pneumonectomy, those who had undergone left pneumonectomies, those who had received radiotherapy prior to the study, those with small cell lung cancer, and those with distant metastases. All the patients included in the study had developed locoregional recurrence after curative right pneumonectomy. In the 23 cases, the histology at recurrence was similar to that of the initial tumor. The staging evaluations that were carried out included the medical history of patients, complete blood counts, physical examination, biochemical tests, bronchoscopy, tomography and chest $X$-rays, ultrasound images of the abdomen, as well as liver, brain, and bone radionuclide scans. Locoregional recurrence was diagnosed using computed tomotherapy, [18F] FDG-PET, and abnormal observations on bronchoscopy. The following criteria of the results for PFT (pulmonary function test) must be considered for tomotherapy: (1) baseline $\mathrm{FEV}_{1}>35 \%$ predicted; (2) diffusion capacity > 35\% predicted; (3) absent baseline hypoxemia (hypoxemia defined as $\mathrm{PaO} 2$ of $\leq 60 \mathrm{mmHg}$ on room air) and/or hypercapnia (hypercapnia defined as $\mathrm{PaCO}_{2}>50 \mathrm{mmHg}$ ).

\section{Treatment planning}

All the target delineation was performed in the Pinnacle treatment planning system (Philips Medical Systems, Bothell, WA). Afterwards, each patient's planning CT scan and the contours were transferred into the helical tomotherapy planning system (Tomotherapy Inc.) for treatment planning. The primary planning target volume (PTVp) associated with the primary tumor gross tumor volume (GTV) had a $10 \mathrm{~mm}$ margin from GTV (Fig. 1). The clinical target volume (CTV) was defined by adding $8 \mathrm{~mm}$ margins around the gross 

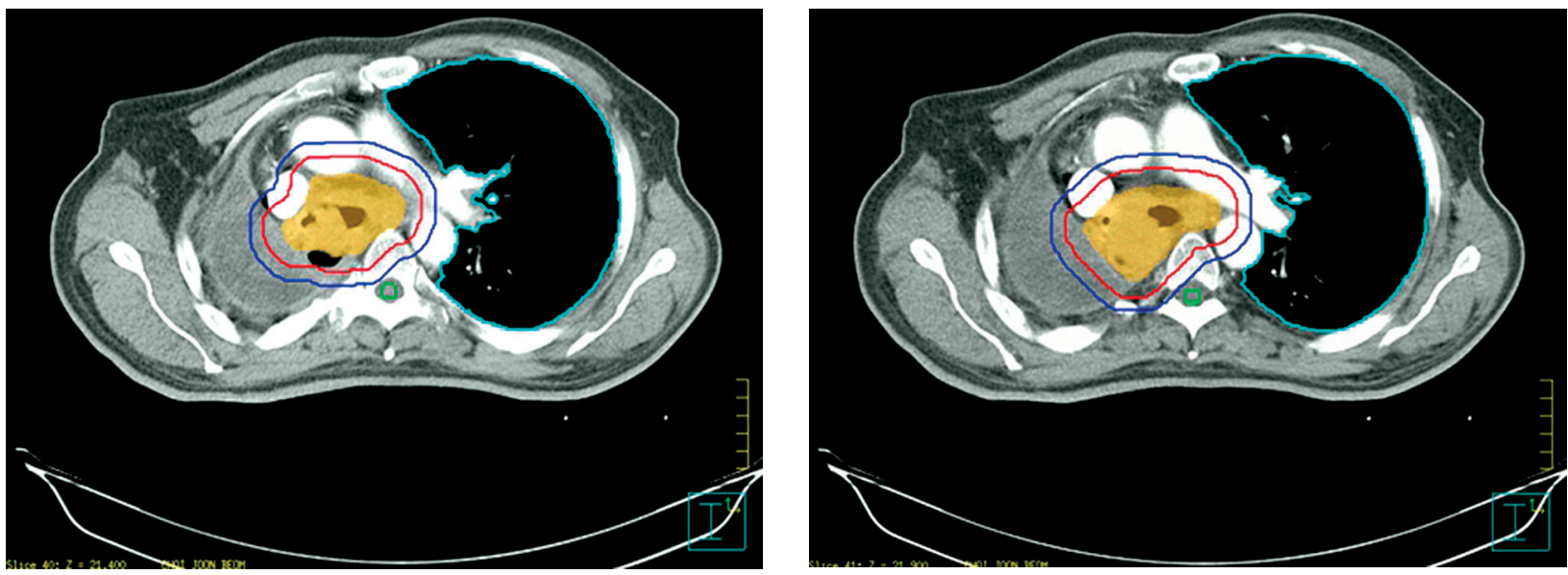

Fig. 1. Primary PTV (red line) and secondary PTV (blue line). The primary planning target volume (PTV) associated with the primary tumor gross tumor volume (GTV) had a $10 \mathrm{~mm}$ margin from the GTV. The CTV were defined by adding $8 \mathrm{~mm}$ margins around the GTV in this patient with adenocarcinoma. By taking tumor motion and setup uncertainty into consideration, the secondary PTV was defined by adding $10 \mathrm{~mm}$ margins around the CTV

tumor volume (GTV). By taking tumor motion and setup uncertainty into consideration, the secondary planning target volume (PTVs) was defined by adding $10 \mathrm{~mm}$ margins around the CTV (Fig. 1). The lungs, esophagus, spinal cord, and heart were contoured for each patient. The major vessels, major airway and other additional structures were contoured only when they were adjacent to the GTV. Treatment plans were generated in the Tomotherapy Hi-Art planning system using $6 \mathrm{MV}$ photons delivered without a flattening filter.

Planning was performed taking into account the field width, pitch, modulation factor, and dose constraints, on the basis of a standardized procedure. The present study used a $5 \mathrm{~cm}$ field width, and a pitch of 0.17 or 0.31 . A modulation factor of 3 was set at the beginning of the optimization process. A binary multi-leaf collimator (MLC) with a leaf width that projected to a $6.25 \mathrm{~mm}$ width at the isocenter was $85 \mathrm{~cm}$ away from the X-ray photon source. In the plans, a longitudinal aperture size of $1.05 \mathrm{~cm}$ or $2.5 \mathrm{~cm}$ was used. The nominal dose rate at the isocenter was $87 \mathrm{cGy} / \mathrm{min}$ (SAD). All plans prescribed 69 Gy delivered in 30 daily fractions to the primary PTV with heterogeneity corrections using the superposition-convolution algorithm, and were optimized to have at least $95 \%$ of the PTV receiving $100 \%$ of the prescription dose, which is in accordance with the American Society of Therapeutic Radiation Oncology (ASTRO)'s white paper.

Target volume coverage took priority over the dose constraints for the OAR (organs at risk) in each case because of the concern for a significant decrease in tumor control probability (TCP) when there are significant subvolumes of cold spots. Helical tomotherapy was used to deliver a dose of 2.3 Gy for a period of 30 days to all patients. As a result, the total radiation received was 69 Gy per patient. Radiation was targeted at both the primary tumor and involved nodal regions. However, elective nodal irradiation was not carried out. Planning imaging involved a thin slice treatment planning CT. Respiratory motion was reduced and normalized using a double vacuum immobilization system.
In order to determine the extent of the GTV, the researchers used PET imaging. The gross tumor volume was defined as the visualization of any gross disease and lymph nodes $\geq 1 \mathrm{~cm}$ on CT. The clinical target volume was defined as the GTV plus a 6- to 8-mm margin (adenocarcinoma: $8 \mathrm{~mm}$; SCC: $6 \mathrm{~mm}$ ). The primary PTV was defined as the gross tumor volume plus a $10 \mathrm{~mm}$ margin to account for tumor motion and setup uncertainty. The secondary PTV was defined as the clinical target volume plus a $10 \mathrm{~mm}$ margin with the same causes for primary PTV.

In addition, the critical structures, including the left lung and the heart, the esophagus, and the spinal cord, were contoured.

The tomotherapy treatment planning system was used to plan treatment. The planning objectives for the tomotherapy plans reflected the following priorities: (1) achieving the plan of dose prescription for the target volume which aimed at a standard deviation (SD) of the dose distribution of no more than $2 \%$ or $3 \%$ of the deliverable dose to PTVp1 and PTVp2; (2) maintaining the maximal dose of the planning spinal cord $\leq 45 \mathrm{~Gy}$; (3) limiting the volume of the left lung receiving 20 Gy (V20) less than 20\%, and mean lung dose < $20 \mathrm{~Gy}$, based on a study of Graham et al. [22]; (4) keeping the volume of the heart receiving 20 Gy $\left(V_{20}\right)$ less than 50\%; (5) limiting the 30\% volume of the esophagus < 60 Gy, 66\% to 55 Gy, and $D_{\max }<75$ Gy. The dose constraints for the lung, the heart, the esophagus, and the spinal cord were corrected for fractionation effects using the linear-quadratic formalism $[23,24]$. The $\alpha / \beta$ value of 3 Gy was selected for the lung and esophagus, 1 Gy for the heart, and 2 Gy for the spinal cord $[25,26]$. The act of repeating the process was required until the objective function was optimized and the treatment planning aims approached the desired goal.

\section{Evaluation of survival and toxicity}

All the patients were followed up for evaluation which included chest CT, chest X-ray, [18F] FDG-PET, and physical examinations. The examination was carried out at intervals 
of three months for a period of two years after the completion of radiotherapy, and thereafter at six-month intervals.

\section{Statistical analysis}

Overall survival (OS) duration was calculated from the first day of tomotherapy to time of death or last follow-up. The Kaplan-Meier method was used for calculation of the survival rates. The Cox proportional hazards model identified the various prognostic factors that might have a significant effect on post-recurrent survival. Several factors such as demographic and clinical characteristics were initially evaluated for their association with postrecurrence survival using univariate analysis by the log-rank test. The relative risk (RR) of death and its 95\% confidence interval (CI) were calculated using multivariate analysis by a Cox regression model. The data were considered as significant when the $p$ value did not exceed 0.05. The Wilcoxon signed rank test was used to test the differences in loss of

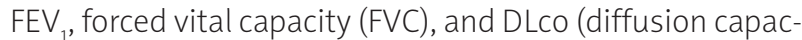
ity, transfer factor) after pneumonectomy and tomotherapy. Two-sided $p$-values $<0.05$ were considered significant. Statistical analyses were performed using SPSS software (release 14.0; SPSS Inc., Chicago, IL, USA).

\section{Results}

\section{Patient characteristics}

There were 4 females and 19 males, with a median age of 65 (range 56-74). Table 1 shows the recurrence T- and $\mathrm{N}$-stage distribution of the patient population according to the (2009) AJCC staging classification. The disease recurrence was stage IIB in 13 (56.5\%), stage IIIA in 6 (26\%), and stage IIIB in 4 (17.5\%). Twelve patients had SCC, and 11 patients had adenocarcinoma. There was no patient with small cell carcinoma. A total of 23 patients received tomotherapy alone without chemotherapy, and were treated with a total dose of 69 Gy at 2.3 Gy per fraction, 5 days per week, for an overall treatment time of 6 weeks.

\section{Results for tomotherapy plans}

The tomotherapy plan for one of the study patients is presented in Fig. 2. This is the most severe case of tomotherapy performed in recurrent cancer at the bronchial stump in our hospital. Among the 23 subjects of this study, the patient had the largest GTV volume $(322.3 \mathrm{cc}$ ) and primary PTV volume $(761.9 \mathrm{cc})$. This case was diagnosed as NSCLCa (adenocarcinoma) stage IIIA (T2N2M0), surgically proven in October 30, 2002. Main surgery was right pneumonectomy and mediastinal lymph node dissection. The patient underwent 6 sessions of chemotherapy of gemcitabine plus cisplatin postoperatively (from December 5 , 2002 to April 11, 2003) and was followed up at 6-month intervals. PET CT and chest CT findings on March 9, 2006 revealed the recurrence at the right bronchial stump site. The patient complained of hoarseness caused by vocal cord paralysis. The second chemotherapy was performed on the patient by changing the chemotherapeutic regimen to paclitaxel plus carboplatin (from March 17, 2006 to January 4, 2007). PET CT findings (May 8, 2008) revealed aggra- vation of the recurrent mass at the stump site and multiple newly developed metastases in upper paratracheal lymph nodes and both (right and left) supraclavicular lymph nodes. Subsequently, the hematooncologist prescribed erlotinib but suspended the medication due to poor compliance with erlotinib. Instead, we implemented radiotherapy. Relapse sites were characterized by both unfavorable tumor geometry and proximity of the tumor to the heart, esophagus, spinal cord, and the single remaining left lung

Table 1. Characteristics of the patients

\begin{tabular}{|c|c|}
\hline Variables & Patients $(n=23)$ \\
\hline Age (SD) & $65( \pm 9.1)$ \\
\hline \multicolumn{2}{|l|}{ Sex, No. (\%) } \\
\hline men & $19(82.6)$ \\
\hline women & $4(17.3)$ \\
\hline \multicolumn{2}{|l|}{$\begin{array}{l}\text { Initial pathologic stage, No. (\%) } \\
\text { (after pneumonectomy) }\end{array}$} \\
\hline 1 & $8(34.8)$ \\
\hline$\|$ & $5(21.7)$ \\
\hline IIIA & $4(17.3)$ \\
\hline$\| \mathrm{IIB}$ & $6(26)$ \\
\hline \multicolumn{2}{|l|}{$\begin{array}{l}\text { ECOG performance scale, No. (\%) } \\
\text { (when recurrence) }\end{array}$} \\
\hline 1 & $15(65.2)$ \\
\hline 2 & $4(17.3)$ \\
\hline 3 & $4(17.3)$ \\
\hline \multicolumn{2}{|l|}{ Weight loss (when recurrence) } \\
\hline$<5 \%$ & $12(52)$ \\
\hline$\geq 5 \%$ & $11(48)$ \\
\hline $\begin{array}{l}\text { Histology, No. }(\%) \\
\text { squamous cell carcinoma } \\
\text { adenocarcinoma }\end{array}$ & $\begin{array}{l}12(52.1) \\
11(47.8)\end{array}$ \\
\hline \multicolumn{2}{|l|}{$\begin{array}{l}\text { Nodal station with disease, No. (\%) } \\
\text { (after pneumonectomy) }\end{array}$} \\
\hline NO & $13(56.5)$ \\
\hline N1 & $5(21.7)$ \\
\hline N2 & $3(13)$ \\
\hline N3 & $2(8.7)$ \\
\hline \multicolumn{2}{|l|}{ Recurrent stage (\%) } \\
\hline IIB & $13(56.5)$ \\
\hline IIIA & $6(26)$ \\
\hline IIIB & $4(17.5)$ \\
\hline $\begin{array}{l}\text { Reason for the pneumonectomy, No. (\%) } \\
\text { direct extension } \\
\text { metastatic lymph node }\end{array}$ & $\begin{array}{c}15(65.2) \\
8(34.7)\end{array}$ \\
\hline $\begin{array}{l}\text { Previous chemotherapy, No. (\%) } \\
\text { neoadjuvant chemotherapy } \\
\text { postoperative adjuvant chemotherapy }\end{array}$ & $\begin{array}{c}6(26) \\
10(43.5)\end{array}$ \\
\hline $\begin{array}{l}\text { Previous radiotherapy, №. (\%) } \\
\text { neoadjuvant radiotherapy } \\
\text { postoperative adjuvant radiotherapy }\end{array}$ & $\begin{array}{c}6(26) \\
10(43.5)\end{array}$ \\
\hline $\begin{array}{l}\text { Gross tumor volume }\left(\mathrm{cm}^{3}\right) \text { (range) } \\
\text { for tomotherapy }\end{array}$ & $254.5(103-322.3)$ \\
\hline $\begin{array}{l}\text { Planning tumor volume }\left(\mathrm{cm}^{3}\right) \text { (range) } \\
\text { for tomotherapy }\end{array}$ & 439 (164-761.9) \\
\hline
\end{tabular}



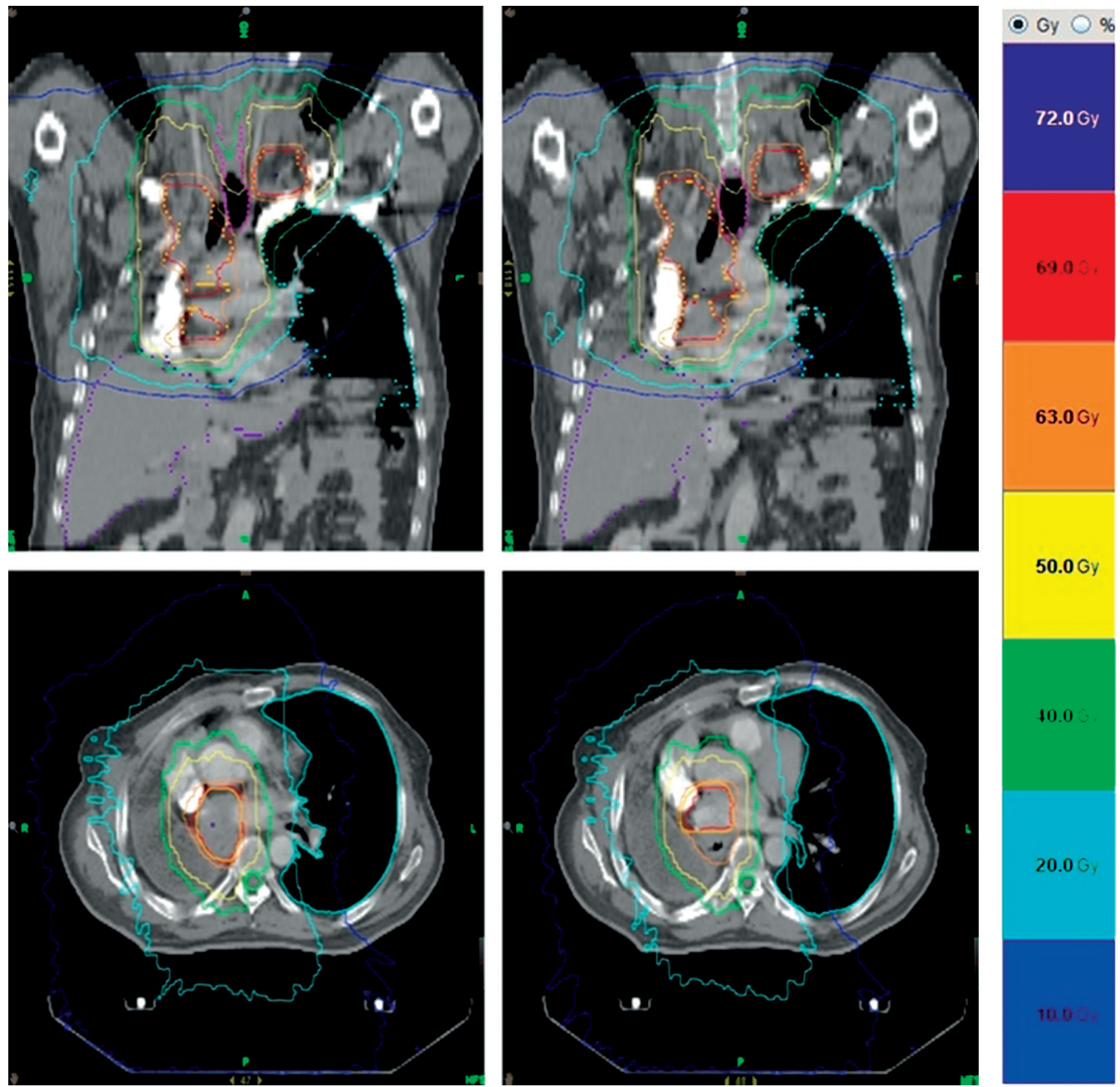

Fig. 2. Example of isodose distribution and drawing of PTV in a single case. Coronal view (upper photo) and axial view (lower photo). This is the most severe case of tomotherapy performed in recurrent cancer at the right bronchial stump in our hospital. Among 23 subjects of this study, the patient had the biggest GTV volume $(322.3 \mathrm{cc})$ and primary PTV volume $(761.9 \mathrm{cc})$

in radiation therapy. Especially, the radiation exposure to both ventricles of the heart had to be reduced. Since the stump recurrent mass maintained a very close distance with the esophagus in addition to the heart, the likelihood of esophageal toxicity was forecasted to be great. Against this clinical background, tomotherapy of intensity-modulated radiation therapy (IMRT) and image-guided radiation therapy (IGRT) was performed.

GTV1 was defined by drawing the right recurrent stump mass, upper paratracheal lymph node metastases and right supraclavicular lymph node metastases. GTV2 was defined by drawing left supraclavicular lymph node metastases. This patient had a GTV volume (GTV1 plus GTV2) of 322.3 cc. The primary PTV was composed of PTVp1 and PTVp2 (Fig. 1). The CTV1 and CTV2 were defined by adding $8 \mathrm{~mm}$ margins around GTV1 and GTV2, respectively. By taking tumor motion and setup uncertainty into consideration, the PTVs1 (582.67 cc) and PTVs2 (179.23 cc) were defined by adding $10 \mathrm{~mm}$ margins around CTV1 and CTV2, respectively (Fig. 1). This patient had a total PTV of $761.9 \mathrm{~cm}^{3}$ occupying a stump recurrent mass and metastatic lymph nodes.
The results of the pulmonary function test before radiation therapy are $\mathrm{FEV}_{1}>700 \mathrm{cc}$, predicted $\mathrm{FEV}_{1}$ value $>40 \%$. Therefore, we decided on high-dose radiation therapy alone by tomotherapy with radical and curative aims. In this case, the $95 \%$ volume of the primary PTV was prescribed to a total dose of 69 Gy in 30 fractions, and 95\% of the secondary PTV was given to a total dose of 54 Gy in 30 fractions. The BED (biologically effective dose) for primary PTV and secondary PTV was $84.87 \mathrm{~Gy}_{10}$ and 63.72 $G y_{10}$, respectively $(\alpha / \beta$ ratio $=10)$. Thus, a simultaneous integrated boost (SIB) was applied to cover several anatomic target sites and with the notion of a treatment strategy of clinical and dosimetric benefits. The treatment was completed in 6 weeks, which corresponded to a moderate shortening of treatment time.

As shown in the dose-volume histogram (DVH) of Fig. 3, a steep incline was observed in the graph of PTVs1 and PTVs2 in the initial period, and then it changed to a gentle slope at the dose range of 55-60 Gy. After a dose of 70 Gy, a steep incline was seen again in the graph. Although 95\% of PTVs1 and PTVs2 was exposed to a dose of 54 Gy, a small volume of PTVs was exposed to a high dose of ra- 


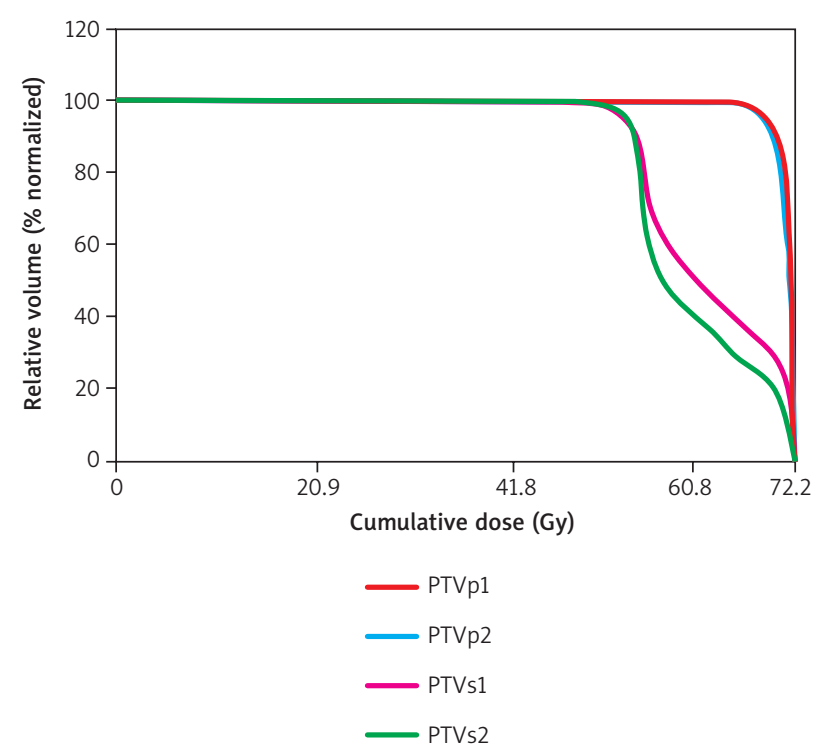

Fig. 3. Example of dose-volume histograms (DVHs) of planning tumor volume (PTV) in the same case presented in Fig. 1 and Fig. 2. As shown in the DVH of Fig. 3, a steep incline was observed in the graph of PTVs1 and PTVs2 in the initial period, and then it changed to a gentle slope at the dose range of 55-60 Gy. After a dose of $70 \mathrm{~Gy}$, a steep incline was seen again in the graph

diation because a 95\% of PTVp1 and PTVp2 was exposed to a dose of 69 Gy at the same time.

The duration of tomotherapy was from December 23, 2009 to February 12, 2010 (52 days). After the treatment, history taking, physical examination, and chest $\mathrm{X}$-ray were performed at a 3-month interval for the first two years. The interval of the next follow-up period was 6 months. Chest $\mathrm{CT}$ and PET CT were conducted to evaluate the response of tomotherapy in January 16, 2013 after finishing tomotherapy. Both chest CT and PET CT showed NED (no evidence of disease). However, the patient abruptly complained about dysphagia, and emergently performed endoscopic biopsy of the distal esophageal mass revealed the pathologic results of adenocarcinoma in March 4, 2013. The patient was on treatment with pemetrexed. PET after pemetrexed revealed markedly improved metabolism of a known metastasis in the distal esophagus $\left(\mathrm{SUV}_{\max } 18.0->8.6\right)$ in May 16, 2013. The maximal dose of the esophagus was 69 Gy due to the recurrent stump mass completely abutting the esophagus. As a result, the patient had to undergo tomotherapy, suffering from esophagitis grade 3. Eventually, the patient overcame the adverse reaction with medical supportive care including nystatin, tetracycline, fentanyl, and 1-week rest. Esophageal stricture has not been found until now as a complication of tomotherapy with a high radiation dose. Limitation of radiation dose exposed to the left lung maintained the $20 \%$ volume of the left lung $<20$ Gy, and mean lung dose < 20 Gy. No radiation pneumonitis was detected. When radical and curative, highdose tomotherapy is applied to patients with recurrent stump lung cancer having only one remaining lung after pneumonectomy, the new guidelines of constraints for only one remaining lung need to be investigated more in

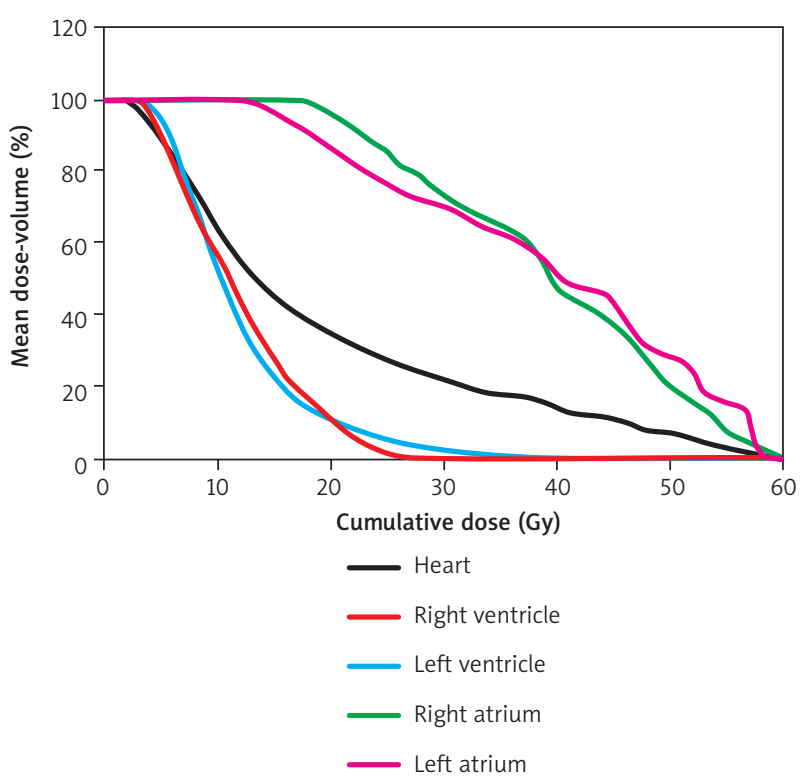

Fig. 4. Mean dose-volume histograms for the heart in the 23 cases of this study. The mean $\mathrm{V}_{45}, \mathrm{~V}_{50}, \mathrm{~V}_{60}$ of the heart were $10.5 \%, 6.5 \%$, $0.2 \%$, respectively. In the heart, a $60 \%$ volume of the left atrium and right atrium was mostly exposed to over $40 \mathrm{~Gy}$. A $50 \%$ of the right ventricle was exposed to an average of 11.3 Gy while, a $50 \%$ of left ventricle was exposed to an average of $10.4 \mathrm{~Gy}$

the future. Although the patient in this case report was the most difficult and complex, very satisfactory clinical results were obtained.

To evaluate the quality of the 23 plans created by our team, the conformal index (CI) and heterogeneity index (HI) were calculated on the basis of the DVHs of the PTV. The Cl of the PTV is the volume enclosed by the prescription isodose line divided by the volume of the PTV. The increased Cl shows a greater volume of the prescription dose delivered outside the PTV. The HI of the PTV was defined using the equation of $\mathrm{D} 5 \% / \mathrm{D} 95 \%$, where $\mathrm{D} 5 \%$ and $\mathrm{D} 95 \%$ correspond to the dose given to $5 \%$ and $95 \%$ of the PTV, respectively. A larger $\mathrm{HI}$ is a sign which suggests a greater dose exceeding the prescription dose, and thus greater dose heterogeneity inside the PTV. The median Cls of PTVp and PTVs in the 23 plans were 0.92 and 0.94 , respectively. During our research on radiotherapy of lung cancer, the poor $\mathrm{Cl}$ of $3 \mathrm{D}$ conformal radiotherapy (3DRTx) was frequently observed in most lung cancers with more irregular, complex, and advanced stage, ranging from 2 to 5 . The tomotherapy improved the $\mathrm{Cl}$ of the PTV, showing more conformal and improved isodose distributions. The median heterogeneity index of PTVp and PTVs in the 23 plans was 1.04 and 1.33, respectively. The tomotherapy also improved the HI of the PTVp including the GTV, compared to the already known data of the HI for 3DRTX, on average ranging from 1.2 to 1.5 . However, in general, the $\mathrm{HI}$ of PTVs including the PTVp increased, compared to the $\mathrm{HI}$ of the PTVp. The reason for the increased HI of PTVs was the planning and irradiation of different targets at different dose levels in a single treatment session in simultaneous integrated boost (SIB)-IMRT used in this study.

The $V_{x}(\%)$ of the left lung and mean lung dose presented as the median, with the range in parentheses, are as 

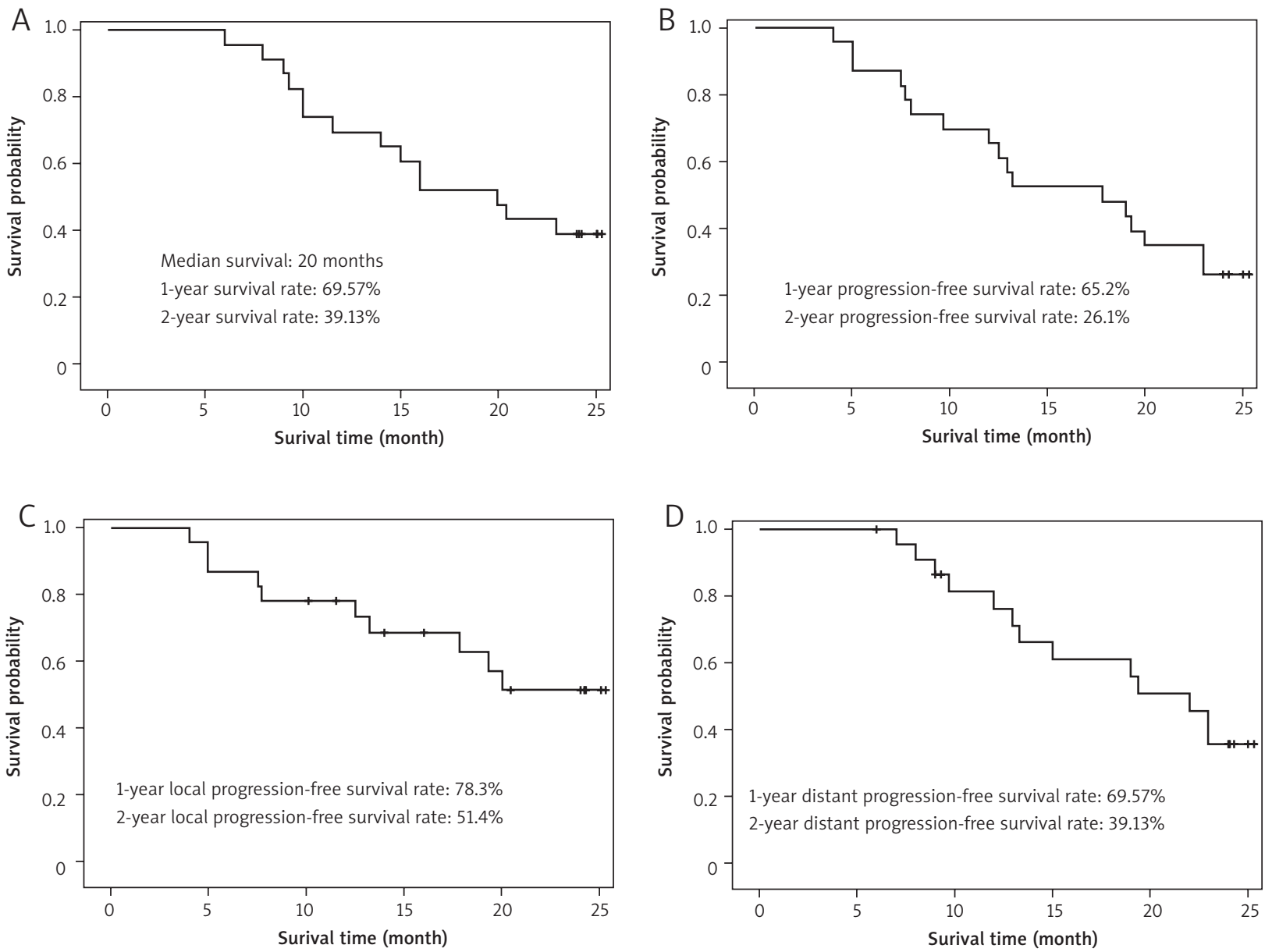

Fig. 5. Overall survival rate and progression-free survival rate. The median follow-up after tomotherapy was 19.86 months (range 6 to 26.5 months). This analysis of treatment outcome was based on the follow-up data available as of February 28, 2014. Overall survival duration was assessed from the first day of tomotherapy to the time of death or last follow-up, and estimated using the Kaplan-Meier method. Median survival was 20 months. The 1-year and 2-year OS rates were $69.5 \%$ and $39.1 \%$, respectively

follows: left lung $V_{5}(\%)$ : 52.35 (21-82), left lung $\bigvee_{10}(\%)$ : 36.20 (16-57), left lung $V_{20}(\%): 14.1$ (11-16), and mean lung dose (Gy): 15.54 (9.7-26). There was a case where the volume of the left lung exposed to low dose radiation increased to $82 \%$. However, no severe radiation pneumonitis was detected. Further evaluation and study of the parameters which have a significant effect on the degree of single remaining left lung sparing are crucial.

The mean $V_{45}, V_{50}, V_{60}$ of the esophagus were $79 \%, 69 \%$, $31 \%$, respectively. The dose delivered to the esophagus was increased. The percentage volume of the esophagus at 55 Gy was 46 (38-71). The maximal dose of the esophagus increased to about $75 \mathrm{~Gy}$ in the case of the stump recurrent mass abutting the esophagus at the level of T5-T7. In this case of the recurrent stump mass abutting the esophagus, tomotherapy was resumed after a week rest period due to esophagitis of grade 3 and then was completed with all required procedures. Consequently, CR (complete response) was decided in the PET study after a 6-month follow-up.

The mean $V_{45}, V_{50}, V_{60}$ of the heart were $10.5 \%, 6.5 \%$, $0.2 \%$, respectively. In the heart, $60 \%$ volume of the left atrium and right atrium was mostly exposed to above $40 \mathrm{~Gy}$. $50 \%$ of the right ventricle was exposed to an average of
11.3 Gy, while $50 \%$ of the left ventricle was exposed to an average of 10.4 Gy (Fig. 4).

\section{Treatment outcome - survival rate and treatment failure}

The median follow-up after tomotherapy was 19.86 months (range 6 to 26.5 months). This analysis of treatment outcome was based on the follow-up data available as of February 28, 2014. Overall survival duration was assessed from the first day of tomotherapy to the time of death or last follow-up, and estimated using the Kaplan-Meier method. Median survival was 20 months. The 1-year and 2-year OS rates were $69.5 \%$ and $39.1 \%$, respectively (Fig. 5).

After tomotherapy, 7 out of 23 patients died within 1 year. Three out of 7 patients died of local progression at a median of 7.5 months, 2 out of 7 patients died of distal progression at a median of 8.9 months, and the other 2 patients died of local progression followed by distant progression at a median of 5 months. Therefore, 1-year OS was $69.5 \%$ (16 out of 23 patients survived) (Fig. 5). The local progression-free survival rate was $78.3 \%$ at 1 year (Fig. 5). The distant progression-free survival rate was $69.57 \%$ at 
1 year (Fig. 5). Total distant metastasis was detected in the brain $(n=1)$, right kidney $(n=1)$, left lung $(n=1)$, and pericardium $(n=1)$.

Two years after tomotherapy, 7 out of 16 survivors died after another year. All of these 7 patients died of distant metastasis. The cause of death of 4 patients was local progression followed by distant progression at a median of 15.5 months. The other 3 patients died of distant progression at a median of 13 months. Therefore, 2-year OS was 39.1\% (9 out of 16 patients survived) (Fig. 5). The local progression-free survival rate was $51.4 \%$ at 2 years (Fig. 3 ). The distant progression-free survival rate was $39.1 \%$ at 2 years (Fig. 5). Total distant metastasis was observed in the liver $(n=1)$, brain $(n=3)$, bone $(n=1)$, multiple lymph nodes $(n=1)$, and adrenal gland $(n=1)$.

\section{Analysis of prognostic factors for overall survival}

The results of the Cox regression analysis are shown in Table 2. The left panel shows the crude hazard ratio (HR) for each variable based on univariate analysis. Compared to the $<65 \mathrm{yr}$ group, the crude HR of mortality for the $\geq 65$ yr group was 3.251 (95\% Cl: $1.1-9.4 ; p=0.03)$. The other variables showed no significant correlation with mortality in the univariate analysis. In the final multiple regression model (the right panel of Table 2), the adjusted HR of mortality for the $\geq 65 \mathrm{yr}$ group was 6.4 (95\% Cl: 1.2-11.2; $p=0.032$ ) after controlling for other explanatory variables. Other predictors selected in the final model for the risk of mortality were ECOG performance status at recurrence, stage at recurrence, and weight loss.

Table 2. Prognostic factors associated with overall survival

\begin{tabular}{|c|c|c|c|c|c|c|}
\hline \multirow[t]{2}{*}{ Prognostic factors } & \multicolumn{3}{|c|}{$\begin{array}{l}\text { Crude hazard ratio } \\
\text { on univariate analysis } \\
(95 \% \mathrm{Cl})\end{array}$} & \multicolumn{3}{|c|}{$\begin{array}{l}\text { Adjusted hazard ratio } \\
\text { on multivariate analysis } \\
(95 \% \mathrm{Cl})\end{array}$} \\
\hline & Crude HR & $95 \% \mathrm{Cl}$ & $P$ Value & Adjusted HR & $95 \% \mathrm{Cl}$ & $P$ Value \\
\hline $\begin{array}{l}\text { Age at recurrence } \\
<65 \text { years } \\
\geq 65 \text { years }\end{array}$ & $\begin{array}{c}1 \\
3.251\end{array}$ & $1.1-9.4$ & 0.03 & $\begin{array}{c}1 \\
6.4\end{array}$ & $1.2-11.2$ & 0.032 \\
\hline $\begin{array}{l}\text { ECOG performance status a } \\
\text { 0-1 } \\
2-4\end{array}$ & $\begin{array}{c}1 \\
2.6\end{array}$ & $0.1-5.9$ & 0.059 & $\begin{array}{c}1 \\
3.7\end{array}$ & $1.13-6.3$ & 0.045 \\
\hline $\begin{array}{l}\text { Symptoms when recurrence } \\
\text { no } \\
\text { yes }\end{array}$ & $\begin{array}{c}1 \\
1.3\end{array}$ & $0.4-3.8$ & $0.35(>0.2)$ & $\begin{array}{c}1 \\
3.1\end{array}$ & $0.07-5.8$ & \\
\hline $\begin{array}{l}\text { Disease-free interval (years) } \\
<1 \text { years } \\
\geq 1 \text { years }\end{array}$ & $\begin{array}{c}1 \\
0.8\end{array}$ & $0.2-8.7$ & 0.09 & $\begin{array}{c}1 \\
0.87\end{array}$ & $0.03-3.34$ & 0.66 \\
\hline $\begin{array}{l}\text { Initial pathologic stage } \\
\text { stage I } \\
\text { stage II } \\
\text { stage III }\end{array}$ & $\begin{array}{c}1 \\
1.7 \\
2.5\end{array}$ & $\begin{array}{l}0.47-2.3 \\
0.33-5.1\end{array}$ & 0.085 & $\begin{array}{c}1 \\
2.52 \\
2.69\end{array}$ & $\begin{array}{c}0.31-4.8 \\
0.45-4.94\end{array}$ & 0.93 \\
\hline $\begin{array}{l}\text { Stage at recurrence } \\
\text { stage IIB } \\
\text { stage IIIA-IIIB }\end{array}$ & $\begin{array}{c}1 \\
1.7\end{array}$ & $0.12-4.1$ & 0.067 & $\begin{array}{c}1 \\
2.31\end{array}$ & $1.62-3.02$ & 0.014 \\
\hline $\begin{array}{l}\text { Sex } \\
\text { female } \\
\text { male }\end{array}$ & $\begin{array}{c}1 \\
0.3\end{array}$ & $0.04-2.41$ & $0.51(>0.2)$ & $\begin{array}{c}1 \\
4.9\end{array}$ & 0.49-9.11 & \\
\hline $\begin{array}{l}\text { Cell type } \\
\text { adenocarcinoma } \\
\text { squamous cell carcinoma }\end{array}$ & $\begin{array}{c}1 \\
1.5\end{array}$ & $0.5-4.3$ & 0.15 & $\begin{array}{c}1 \\
2.14\end{array}$ & $0.95-3.23$ & 0.27 \\
\hline $\begin{array}{l}\text { Neoadjuvant chemotherapy } \\
\text { yes } \\
\text { no }\end{array}$ & $\begin{array}{c}1 \\
0.98\end{array}$ & $0.7-1.8$ & $0.27(>0.2)$ & $\begin{array}{c}1 \\
5.6\end{array}$ & $0.75-10.8$ & \\
\hline $\begin{array}{l}\text { Postoperative adjuvant cher } \\
\text { yes } \\
\text { no }\end{array}$ & $\begin{array}{c}1 \\
0.5\end{array}$ & $0.1-1.5$ & $0.49(>0.2)$ & $\begin{array}{c}1 \\
2.36\end{array}$ & $0.71-3.68$ & \\
\hline $\begin{array}{l}\text { Weight loss } \\
\text { none } \\
\geq 5 \%\end{array}$ & $\begin{array}{c}1 \\
2.1\end{array}$ & $0.7-6.1$ & 0.096 & $\begin{array}{c}1 \\
4.38\end{array}$ & $1.86-7.3$ & 0.025 \\
\hline
\end{tabular}

The Cox proportional-hazards model was used to identify prognostic factors associated with overall survival. All factors with a univariate significance of $p<0.20$ were entered in the multivariate model. The proportional-hazards assumption was checked using a test on Schoenfeld residuals and was found to be valid for all factors investigated. 
Table 3. Variable changes in FEV ${ }_{1}$, FVC, and DLco after pneumonectomy and tomotherapy according to the age of the patients at tomotherapy

\begin{tabular}{|c|c|c|c|}
\hline Pneumonectomy & $\begin{array}{c}\Delta \mathrm{FEV}_{1} \\
(\% \text { pred) } \\
\text { (mean } \pm \mathrm{SD})\end{array}$ & $\begin{array}{c}\Delta \text { FVC } \\
(\% \text { pred }) \\
(\text { mean } \pm \text { SD) }\end{array}$ & $\begin{array}{c}\Delta \text { DLco } \\
(\% \text { pred) } \\
\text { (mean } \pm \text { SD) }\end{array}$ \\
\hline $\begin{array}{l}<65 \text { years } \\
(n=19)\end{array}$ & $\begin{array}{c}31.7 \pm 16 \\
(P=0.043)\end{array}$ & $\begin{array}{c}31 \pm 24 \\
(P=0.039)\end{array}$ & $\begin{array}{c}33.8 \pm 24 \\
(P=0.042)\end{array}$ \\
\hline $\begin{array}{l}\geq 65 \text { years } \\
(n=4)\end{array}$ & $\begin{array}{c}21.9 \pm 11 \\
(P=0.037)\end{array}$ & $\begin{array}{c}23.7 \pm 20 \\
(P=0.047)\end{array}$ & $\begin{array}{c}26.7 \pm 24 \\
(P=0.035)\end{array}$ \\
\hline Tomotherapy & $\begin{array}{c}\Delta \text { FEV }_{1} \\
(\% \text { pred }) \\
(\text { mean } \pm \text { SD })\end{array}$ & $\begin{array}{c}\Delta \text { FVC } \\
(\% \text { pred }) \\
(\text { mean } \pm \text { SD })\end{array}$ & $\begin{array}{c}\Delta \text { DLco } \\
(\% \text { pred }) \\
\text { (mean } \pm \text { SD) }\end{array}$ \\
\hline $\begin{array}{l}<65 \text { years } \\
(n=19)\end{array}$ & NS & NS & NS \\
\hline $\begin{array}{l}\geq 65 \text { years } \\
(n=4)\end{array}$ & NS & NS & NS \\
\hline
\end{tabular}

\section{Variable changes in PFT (pulmonary function test)}

There was a significant difference between preoperative and postoperative FEV,$F V C$, and DLco values for right pneumonectomy in both age groups $(p<0.05)$. However, there was no significant difference between pre tomotherapy and post tomotherapy for the reductions of pulmonary function test in both age groups (Table 3).

\section{Complications}

Various grades of radiation pneumonitis were observed among 7 patients (30\%). One of the patients included in the study had grade 3 radiation pneumonitis and was treated using steroid therapy. The patient recovered. Within 6 months after the radiation treatment, 3 patients died of pulmonary complications. One patient died of bacterial pneumonia, while 2 died of radiation pneumonitis. Nine patients (39\%) experienced radiation esophagitis after the treatment. Five patients suffered grade 3 esophagitis and therefore required fluid support during radiation therapy.

\section{Discussion}

Lung cancer is considered to be the leading cause of cancer-related deaths in the world today. The discussion about NSCLC recurrence after pneumonectomy has been rather peripheral [2]. The literature on the effectiveness of using radiation therapy alone in treating recurrent lung cancer among patients who have undergone right pneumonectomy is even scarcer. The scarcity is the reason why a uniform policy has not been put in place to tackle these issues in the world today. Many of the reports include studies that were carried out over periods of many years and involve multiple therapeutic and diagnostic approaches. Moreover, it is to be noted that most of the studies do not have information on treatment and expected outcomes among patients who have stump recurrences. One of the main reasons why the survival rate among recurrence patients is low, even after subsequent radiation therapy, is that post-operative irradiation is delayed. In most cases, it is delayed until such a time when recurrence has already occurred. Various studies have pointed out that post-oper- ative irradiation is effective, especially if it is directed and confined to the stump site [27-33]. In spite of the fact that this clinical investigation only involved 23 patients, it was carried out over a relatively short period of time and endeavored to ascertain the effectiveness of tomotherapy as a sole treatment.

Despite the use of pneumonectomy and other resection procedures for the treatment of NSCLC, up to $30 \%$ of the patients experience recurrence. Locoregional recurrence is very important for the attainment of overall survival and cure for NSCLC patients. As a result of this, subsequent treatment is required. Although reoperation is adopted for the treatment of some patients, the procedure is very limited. The two main modes of treatment for locoregional recurrence are chemotherapy and radiotherapy. Many different retrospective studies have documented that the median overall survival for such patients is between 10 and 20 months. There are many prognostic factors, which have diverse impacts on the OS. They include where the recurrence has occurred, the objective of the treatment (palliative or curative), disease-free interval, and the incorporation of chemotherapy.

Other studies have suggested that patients who have undergone resection after recurrence have a rate of survival that is comparable to that of those who have just been diagnosed with NSCLC. The observation is made when the two groups of patients receive curative radiotherapy or chemotherapy. For the attainment of cure, the researchers have pointed out that patients who have suffered from NS$\mathrm{CLC}$ recurrence should be offered aggressive therapy. However, one of the main concerns of administering curative radical aggressive radiotherapy to NSCLC recurrence patients is the impact of such treatment on their pulmonary functions. In the recurrent case, the lung function of the patients was already compromised because of their first operation after they were initially diagnosed with NSCLC. Even if the pulmonary function of the patients is normal after right pneumonectomy, there is always the likelihood that salvage radiation will interfere with lung function.

The current study had two major objectives: (1) to determine the effectiveness of tomotherapy in radiation dose escalation for the treatment of inoperable recurrent non-small cell lung cancer at the stump site after right pneumonectomy; (2) to determine the effectiveness of tomotherapy in reducing radiation exposure to the rightshifted heart and to the single remaining left normal lung for the treatment of inoperable recurrent non-small cell lung cancer at the stump site after right pneumonectomy.

Our results of median survival of 20 months and $39.13 \%$ survival rate within 2 years were comparable to the findings of other investigators. For instance, Kagami et al. [30] documented that the median survival and 2-year overall survival rate among 32 locoregional recurrent NSCLC patients were 14 months and $28.1 \%$, respectively. Most authors found that patients who were treated with curative intent of a dose of more than 50 Gy had better health outcomes than their counterparts who had received lower palliative doses. In the retrospective study of Jeremic et al. $[9,10]$, which included 61 patients who were irradiated with either curative intent (tumor dose (TD) 55-60 Gy 
in 26-30 fractions) $(n=42)$ or palliative intent (TD 30 Gy in 10 fractions) ( $n=19)$, the median survival and 2-year survival rate of patients treated with radical aims were 18 months and $36 \%$, respectively, compared to 7 months and $11 \%$ for patients treated with palliative aims. The survival results were significantly different between curative intent and palliative intent $(p=0.000)$. Foo et al. [31] also reported that median survival of patients who were treated with radical radiotherapy for curative purposes was 26 months. On the other hand, those who were treated with lower doses for palliative purposes had a median survival of 10.5 months $(p=0.025)$. Curran et al. [32], who compared the use of radiotherapy among patients who were resected with those who had not received surgical treatment, used a radiation dose of 56 Gy for those suffering from recurrent disease and 59 Gy for newly diagnosed NSCLC. The median survival rate for the patients in both groups was 12 months, while the 2-year survival rate was $22 \%$ and $26 \%$ among the recurrent and newly diagnosed patients respectively ( $p$ value was non-significant). When the patients who had bronchial stump recurrence were compared to those having lesions in the chest wall or lymph nodes, a significant difference was evident. Patients with bronchial stump lesions had a median survival of 36 months, compared to the patients with nodal or chest wall recurrence (median survival of 9 and 7 months, respectively). In this study, the investigators concluded that aggressive radiotherapy should be offered to those patients who have suffered locoregional recurrence after resection and those newly diagnosed with NSCLC. Cai et al. [33] carried out a study to draw comparisons between newly diagnosed NSCLC patients and those who had locoregional recurrence after resection. They suggested that locoregional recurrence patients should receive aggressive chemotherapy and radiotherapy. In their study, they did not find any significant differences in the overall rate of survival among stage I to stage III patients suffering from newly diagnosed disease and recurrence patients. These findings suggest that patients with postresection recurrent NSCLC should be treated as aggressively as those with newly diagnosed disease.

During radiotherapy for bronchial stump recurrence of lung cancer, the distances of the right atrium and left atrium get close to the GTV. For this reason, $60 \%$ volume of the left atrium and right atrium was mostly exposed to $40 \mathrm{~Gy}$. The results from physical analysis of dosimetric factors for the heart are as follows: (1) The percentage volume of the heart at 40 Gy was 13.7 (0.0-26.7). The effect of radiation on the heart was insignificant. (2) The radiation dose exposed to $60 \%$ volume of the right atrium and left atrium increased to $40 \mathrm{~Gy}$ each, compared to the radiation dose exposed to $60 \%$ volume of the left ventricle of mean 9.3 Gy. To sum up, exposed radiation doses are $60 \%$ volume of left ventricle: mean $9.3 \mathrm{~Gy}$; $60 \%$ volume of right ventricle: mean $9.7 \mathrm{~Gy}$; $60 \%$ volume of left atrium: mean 40 Gy; and 60\% volume of right atrium: mean 40 Gy (Fig. 4). Radiation damage to the heart muscle is called cardiomyopathy. It generally affects the left ventricle (chamber) more than other parts of the heart, causing it to be stiff and less responsive to changes. In patients treated for
non-Hodgkin's lymphoma, doses > 40 Gy to the mediastinum are associated with significantly increased risk of chronic heart failure and myocardial infarction [34]. Doses should be $<40$ Gy for $<25 \%$ of cardiac volume and 30 Gy for larger volumes [35]. However, in this study, the mean of $60 \%$ volume of the left ventricle was $9.3 \mathrm{~Gy}$, and the mean of $25 \%$ volume of the heart was 27 Gy. Therefore, serious cardiac complications were not detected at almost low dose to the left ventricle. Reasons for the increased amount of radiation exposure to the right atrium and the left atrium than the left ventricle are as follows: (1) right-sided shift of the heart after right pneumonectomy; surface characteristics of the chambers of the heart determine that the right atrium forms the right border of the heart, part of the base, and part of the sternocostal surface, and the left atrium forms the posterior surface (base) of the heart, lying entirely within the boundaries of the oblique sinus of the pericardial sac; (2) the recurrent mass at the bronchial stump close to the left atrium and right atrium because of superior location of both atria compared to the other three chambers of heart at the level of the carina.

Researchers have noted that pneumonectomy can cure NSCLC because it is a relatively safe procedure. However, when compared to sublobar or lobar resection, it has high mortality and morbidity rates. Various researchers are of the opinion that the operation scale, as opposed to neoadjuvant therapy, is the main contributor to postoperative mortality and morbidity rates [36]. Additionally, most patients suffering from lung cancer develop recurrence after successful resection. The recurrence can either be distant metastasis or locoregional recurrence. Between 10 and $35 \%$ of NSCLC patients develop isolated recurrence, while more than $50 \%$ of such patients develop both distant metastasis and locoregional recurrence. As already mentioned earlier in this paper, there are quite a number of studies that have documented findings in this regard. The findings from the current study will be seminal in informing policy with regard to effective treatment of inoperable recurrent NSCLC among patients who have undergone surgery. Effective treatment involves both tumor dose escalation and cardiac and pulmonary dose reduction. Penagaricano and colleagues [37] note that tomotherapy is a radiation therapy technique that allows for conformal distribution of the dose to the volume that is targeted while at the same time reducing irradiation of normal tissues. In this regard, it is a technique that can reduce the toxicity of normal tissue and allow the escalation of the dose to the targeted volume, thus culminating in higher local control rates. The findings of the current study will go a long way in complementing such studies in the field. Presently, there are no conclusive guidelines on the optimal dose for the treatment of inoperable NSCLC. Lack of such conclusive guidelines is especially notable in cases of bronchogenic carcinoma. It has also been observed that local control improves with an increase in the dose of radiation [38, 39]. Nevertheless, the optimal dose has been traditionally set from 60 to 70 Gy mainly because of the nearness of other structures to tumors. This paper describes the outcomes and characteristics of 23 patients 
with bronchial stump recurrence after curative right pneumonectomy who received subsequent tomotherapy treatment. The 23 patients whose data were used in the study represent a sample of the patients that the researchers come across in their daily professional endeavors.

In summary, our study was conducted with a focus on the special clinical situations of locally advanced recurrent bronchial stump cancer after right pneumonectomy. In these cases, we were faced with difficulties in the choice of treatment method. Challenges we had to overcome in treatment were the inoperability due to the anatomical complexity of the heart and esophagus close to recurrent tumor and the very wide range of recurrent disease from the bronchial stump to the supraclavicular lymph node. Because of the large disease volume with which chemotherapy alone could not deal, we should consider palliative radiotherapy at first. However, we changed the palliative aim to the curative, radical aim because we have the state-of-the-art tomotherapy which is equipped with intensity-modulated radiotherapy and image-guided radiotherapy. Thus, we set the target volume of tomotherapy to the lesions including all of the recurrent sites presented in the patient's body, and prescribed the high dose of 69 Gy fractionated by 30 times to the target volume. The most important points on which we put the emphasis in IMRT planning were the following: the cardiac toxicity and esophagitis, the radiation pneumonitis of the single remaining left lung after right pneumonectomy, the high radiation dose distributed to the recurrent mass and the high risk area around it. It was fortunate that tomotherapy had the potential for further escalating the dose to the large and complex target volume with better sparing of the esophagus, left ventricle and left lung in the present tomotherapy planning. We report excellent survival rates after the fractionated IMRT and IGRT using tomotherapy. The survival data which we analyzed statistically were median survival, 1-year overall survival, 2-year overall survival, local progression-free survival, and distant progression-free survival; they showed encouraging results comparable or superior to other authors' survival data already presented. The dose escalation may have relevance for improved survival. Despite some patients showing radiation-induced morbidity, our method was relatively well tolerated, and the cumulative acute and late side-effect rates were considered acceptable. Therefore tomotherapy may be considered for not only symptomatic palliation but also improving survival of locally advanced right recurrent bronchial stump cancer, and patients with recurrent NSCLC after surgery should not be treated any less aggressively than those with newly diagnosed disease. We will be able to recommend appropriate dose scheduling guidelines for tomotherapy based on a belief founded in this experience. Tomotherapy is gradually being incorporated into the clinical practice of lung cancer, and several questions regarding its safety and superiority can be answered by our results. On the basis of these results, we currently offer fractionated high dose tomotherapy to patients with a reasonable performance status (Karnofsky performance status $>70$ ) and who after multidisciplinary evaluation are expected to benefit from control of the targeted lesion.
However, we think that future prospective trials with great numbers of patients and longer follow-up are required.

The authors declare no conflict of interest.

\section{References}

1. Hung JJ, Jeng W-J, Hsu W-H, Chou T-Y, Huang B-S, Wu Y-C. Predictors of death, local recurrence, and distant metastasis in completely resected pathological stage-I non-small-cell lung cancer. J Thorac Oncol Off Publ Int Assoc Study Lung Cancer 2012; 7: 1115-23.

2. Maeda R, Yoshida J, Hishida T, Aokage K, Nishimura M, Nishiwaki Y, Nagai K. Late recurrence of non-small cell lung cancer more than 5 years after complete resection: incidence and clinical implications in patient follow-up. Chest 2010; 138: 145-50.

3. Kumar P, Herndon J 2nd, Langer M, et al. Patterns of disease failure after trimodality therapy of nonsmall cell lung carcinoma pathologic stage IIIA (N2). Analysis of Cancer and Leukemia Group B Protocol 8935. Cancer 1996; 77: 2393-99.

4. Gail MH, Eagan RT, Feld R, et al. Prognostic factors in patients with resected stage I non-small cell lung cancer. A report from the Lung Cancer Study Group. Cancer 1984; 54: 1802-13.

5. Asamura H, Nakayama $H$, Kondo $H$, Tsuchiya R, Shimosato $Y$, Naruke T. Lymph node involvement, recurrence, and prognosis in resected small, peripheral, non-small-cell lung carcinomas: are these carcinomas candidates for video-assisted lobectomy? J Thorac Cardiovasc Surg 1996; 111: 1125-34.

6. Kato H. Photodynamic therapy for lung cancera review of 19 years' experience. J Photochem Photobiol B 1998; 42: 96-9.

7. Kato H, Furukawa K, Sato M, et al. Phase ii clinical study of photodynamic therapy using mono-L-aspartyl chlorin e 6 and diode laser for early superficial squamous cell carcinoma of the lung. Lung Cancer Amst Neth 2003; 42: 103-11.

8. Furukawa K, Kato H, Konaka C, Okunaka T, Usuda J, Ebihara Y. Locally recurrent central-type early stage lung cancer $<1.0 \mathrm{~cm}$ in diameter after complete remission by photodynamic therapy. Chest 2005; 128: 3269-75.

9. Jeremic B, Bamberg M. External beam radiation therapy for bronchial stump recurrence of non-small-cell lung cancer after complete resection. Radiother Oncol J Eur Soc Ther Radiol Oncol 2002; 64: 251-57.

10. Jeremic B, Shibamoto $Y$, Milicic B, et al. External beam radiation therapy alone for loco-regional recurrence of non-small-cell lung cancer after complete resection. Lung Cancer Amst Neth 1999; 23: 135-42.

11. Jansen JP, Brutel de la Rivière A, Alting MP, Westermann CJ, Bergstein PG, Duurkens VA. Post pneumonectomy syndrome in adulthood. Surgical correction using an expandable prosthesis. Chest 1992; 101: 1167-70.

12. Sugarbaker DJ, Jaklitsch MT, Bueno R, et al. Prevention, early detection, and management of complications after 328 consecutive extrapleural pneumonectomies. J Thorac Cardiovasc Surg 2004; 128: 138-46.

13. Smulders SA, Holverda S, Vonk-Noordegraaf A, et al. Cardiac function and position more than 5 years after pneumonectomy. Ann Thorac Surg 2007; 83: 1986-92.

14. Fernandez FG, Force SD, Pickens A, Kilgo PD, Luu T, Miller DL. Impact of laterality on early and late survival after pneumonectomy. Ann Thorac Surg 2011; 92: 244-49.

15. Mandraffino G, Dalbeni A, Paunovic N, Mormina EM and Imbalzano E. Radiation-induced heart and vessel atherosclerosis disease. Int J Cardiol 2014; 44: 711-14.

16. Apter S, Shemesh J, Raanani P, et al. Cardiovascular calcifications after radiation therapy for Hodgkin lymphoma: computed tomography detection and clinical correlation. Coron Artery Dis 2006; 17: $145-51$.

17. Gyenes G, Rutqvist LE, Liedberg A, Fornander T. Long-term cardiac morbidity and mortality in a randomized trial of pre- and postoper- 
ative radiation therapy versus surgery alone in primary breast cancer. Radiother Oncol J Eur Soc Ther Radiol Oncol 1998; 48: 185-90.

18. Martinou M, Gaya A. Cardiac complications after radical radiotherapy. Semin Oncol 2013; 40: 178-85.

19. Goddu SM, Chaudhari S, Mamalui-Hunter M, et al. Helical tomotherapy planning for left-sided breast cancer patients with positive lymph nodes: comparison to conventional multiport breast technique. Int J Radiat Oncol Biol Phys 2009; 73: 1243-51.

20. Donovan EM, Yarnold JR, Adams EJ, Morgan A, Warrington AP, Evans PM. An investigation into methods of IMRT planning applied to breast radiotherapy. Br J Radiol 2008; 81: 311-22.

21. Caudrelier JM, Morgan SC, Montgomery L, Lacelle M, Nyiri B, Macpherson M. Helical tomotherapy for locoregional irradiation including the internal mammary chain in left-sided breast cancer:dosimetric evaluation. Radiother Oncol 2009; 90: 99-105.

22. Graham MV, Purdy JA, Emami B, Hams W, Bosch W, Lockett MA, Perez CA. Clinical dose-volume histogram analysis for pneumonitis after 3D treatment for non-small cell lung cancer (NSCLC). Int J Radiat Oncol Biol Phys 1999; 45: 323-9.

23. Maciejewski B, Taylor JM, Withers HR. Alpha/beta value and the importance of size of dose per fraction for late complications in the supraglottic larynx. Radiother Oncol 1986; 7: 323-6.

24. Lebesque JV, Keus RB. The simultaneous boost technique: The concept of relative normalized total dose. Radiother Oncol 1991; 22: 45-55.

25. Van Dyk J, Mah K, Keane TJ. Radiation-induced lung damage: Dose-time-fractionation considerations. Radiother Oncol 1989; 14: 55-69.

26. Joiner MC, Van der Kogel AJ. The linear-quadratic approach to fractionation and calculation of isoeffect relationships. In: Steel GG, editor. Basic clinical radiobiology. 1st ed. London: Arnold; 1997 106-123.

27. Leung J, Ball D, Worotniuk T, Laidlaw C. Survival following radiotherapy for post-surgical locoregional recurrence of non-small cell lung cancer. Lung Cancer Amst Neth 1995; 13: 121-27.

28. Tada T, Fukuda H, Nakagawa K, Matsui K, Hosono M, Takada Y, Inoue $Y$. Non-small cell lung cancer: radiation therapy for locoregional recurrence after complete resection. Int J Clin Oncol 2005; 10: 425-28.

29. Steger V, Spengler W, Hetzel J, et al. Pneumonectomy: calculable or non-tolerable risk factor in trimodal therapy for Stage III nonsmall-cell lung cancer? Eur I Cardio-Thorac Surg Off J Eur Assoc Cardio-Thorac Surg 2012; 41: 880-85.

30. Kagami Y, Nishio M, Narimatsu N, Mjoujin M, Sakurai T, Hareyama M, Saito A. Radiotherapy for locoregional recurrent tumors after resection of non-small cell lung cancer. Lung Cancer Amst Neth 1998; 20: 31-5.

31. Foo K, Gebski V, Yeghiaian-Alvandi R, Foroudi F, Cakir B. Outcome following radiotherapy for loco-regionally recurrent non-small cell lung cancer. Australas Radiol 2005; 49: 108-12.

32. Curran Jr WJ, Herbert SH, Stafford PM, et al. Should patients with post-resection locoregional recurrence of lung cancer receive aggressive therapy? Int J Radiat Oncol Biol Phys 1992; 24: 25-30.

33. Cai X-W, Xu L-Y, Wang L, et al. Comparative survival in patients with postresection recurrent versus newly diagnosed non-smallcell lung cancer treated with radiotherapy. Int I Radiat Oncol Biol Phys 2010; 76: 1100-5.

34. Moser EC, Noordijk EM, van Leeuwen FE, et al. Long-term risk of cardiovascular disease after treatment for aggressive non-Hodgkin lymphoma. Blood 2006; 107: 2912-9.

35. Stewart JR, Fajardo LF, Gillette SM, et al. Radiation injury to the heart. Int J Radiat Oncol Biol Phys 1995; 31: 1205-11.

36. Ng T, Birnbaum AE, Fontaine JP, Berz D, Safran HP and Dipetrillo TA. Pneumonectomy after neoadjuvant chemotherapy and radiation for advanced-stage lung cancer. Ann Surg Oncol 2010; 17: 476-82.

37. Peñagarícano JA, Chao M, Van Rhee F, Moros EG, Corry PM, Ratanatharathorn V. Clinical feasibility of TBI with helical tomotherapy. Bone Marrow Transplant 2011; 46: 929-35.

38. Armstrong J, McGibney C. The impact of three-dimensional radiation on the treatment of non-small cell lung cancer. Radiother Oncol J Eur Soc Ther Radiol Oncol 2000; 56: 157-67.
39. Kong F-M, Ten Haken RK, Schipper MJ, et al. High-dose radiation improved local tumor control and overall survival in patients with inoperable/unresectable non-small-cell lung cancer: long-term results of a radiation dose escalation study. Int I Radiat Oncol 2005; 63: 324-33.

\section{Address for correspondence}

\section{Hong Seok Jang}

Department of Radiaton Oncology

Medical College

Catholic University

St. Mary's Hospital

Seoul, South Korea

e-mail: drjangrt@hotmail.com

Submitted: 6.04 .2014

Accepted: 6.08 .2014 